\title{
13. The Digital Archive and Catalogues of the Vanuatu Cultural Centre: Overview, Collaboration and Future Directions
}

\author{
William H. Mohns
}

The Vanuatu Cultural Information Network (VCIN) is an on-going initiative of the Vanuatu Cultural Centre (VCC) to organise, manage and protect its digital archives. ${ }^{1}$ These archives include those of Vanuatu's National Library; Public Library; National Museum; National Photo, Film and Sound Archive (NFFSA); Vanuatu National Heritage Register; Women's Culture Program; Young People's Program; Traditional Resource Management Program; and Sand Drawing Project. Together these sections comprise the Vanuatu Cultural Centre.

The VCIN, known internally as 'the database project', is a collaborative process among all sections of the VCC that began in December 2004 with the placement of a Canadian CUSO volunteer (the author) at VCC and was managed by a 'database committee' of representatives of the various sections of the Cultural Centre. The project is indebted to, and builds on, previous work in cataloguing and documentation at VCC.

This paper focuses on six aspects of the Vanuatu Cultural Information Network: 1. vision and guiding principles; 2. primary components of the project; 3. impacts of the project; 4 . unique features and innovations; 5 . role of researchers; and 6. planned and potential future directions.

\section{Vision and guiding principles for the VCIN}

\section{Vision}

The vision of the VCIN is to make it easier to find information in the Cultural Centre Archives, where a particular individual has the right to access the information, and to preserve the knowledge held in the archives (in the form of catalogue records and digital objects in the archives) through local and offsite backups.

\footnotetext{
1 The author and the Vanuatu Cultural Centre would like to thank CUSO-VSO, AusAID, the U.S. Ambassador's Fund for Cultural Preservation and the Baillie Family for their support of this project.
} 
Central to the first part of this vision is providing a single platform that will connect information across the various sections of the Cultural Centre. For a given cultural event in Vanuatu or for a given village or language group, there may be relevant information in different forms of media spread throughout the archives of the different sections of the VCC. For example, there may be relevant photos, audio and video recordings in the NFFSA; artefacts in the National Museum; text, audio and video in the archives of the National Heritage Register; as well as books and newspaper articles in the National Library.

A goal of the VCIN is to be able to understand how all of these items are connected - to each other, to language, to individual people and to place. As a result, if a user researches a particular cultural ceremony, for example, they will not only find all publicly-accessible information in the archives, but it will also be clear to them how the various objects in the archives are connected. The user will be able to see, for example, that an object that is in the National Museum was used in a particular ceremony that was recorded by the National Photo, Film and Sound Archive (NFFSA), and that documentation on this ceremony is available from the National Library. Moreover, the user should be able to view digitised photographs of the object from the Museum collections, read relevant electronic documents from the National Library, listen to audio recordings and view photographs and video footage of the ceremony from the NFFSA. As a result the user will be able to understand the museum object in a more appropriate cultural context. In addition, they will be able to navigate through the archives in this way in any of Vanuatu's three official languages: Bislama, French and English.

This vision for the VCIN is reflected in the following six guiding principles: make it easier to find things; respect tabu restrictions; understand social and spatial connections; function trilingually and translingually; be easy to use and accessible; and integrate digital content with the catalogue records.

\section{Make it easier to find things}

This initiative is about making it easier to locate holdings in the Cultural Centre Archives: easier to find books, artefacts, information about cultural and historical sites, photographs, videos and audio recordings, and traditional knowledge. At the same time this system must respect the structure of tabu restriction that is critical to the integrity and functioning of the archives.

\section{Respect tabu restrictions of the archives}

The VCIN incorporates differing levels of secured access to the objects and information stored in the network, respecting and reinforcing the existing access structures employed by the Cultural Centre. As such, the VCIN will only make 
it easier to find things that one has a right to access, and therefore has differing levels of secured access to the information stored in the network. Currently the system relies on sixteen different levels of tabu restriction based on gender, family, village, nasara and island.

\section{Understand social and spatial connections}

We want to create a system that understands the connections between the items in the archives, and the people, places, languages and cultures associated with them. In effect the goal is to be able to map these social and spatial contexts and relationships into digital relationships.

\section{Arrange trilingual and translingual connections}

The typical user of the archives is competent in multiple languages and wants to be able to access any material related to their interests, regardless of the language of either the archived object or its catalogue record (its metadata). As such, the VCIN needs to be trilingual in the sense that the user can choose to navigate the archives in any of the three official languages, but also trilingual and translingual in that the user can find what they want in the archives by entering search terms in any of the three languages and find what they are looking for without the item necessarily having to be fully catalogued in the three languages (due to the time constraints and expertise that is required to do so).

\section{Easy-to-use and accessible interfaces for cataloguing and searching}

The system needs to facilitate greater access to the archives, rather than be a barrier, regardless of the computer skills of the user. It must be easy to use for both the cataloguers and for those searching the catalogues, and to be appropriate for people with differing computer and language skills.

\section{Fully integrate and manage our digital archives with the catalogue records}

The system needs to integrate our digital archives with the catalogue information so that the digital objects (full-text document, photo, audio and video recordings) are directly accessible to users alongside the object's detailed catalogue record. Additionally, from a system management perspective, these digital objects and their catalogue records need to be well-organised, easy to manage, and easy to backup. This is a key part of both the Vanuatu Cultural Information Network and of the broader conservation strategy for the archives. 


\section{Primary components of the project}

The work of realising the vision of the VCIN can be broken down into four main areas of work: cataloguing, digitisation, developing or acquiring appropriate software, and the development of a cultural thesaurus.

\section{Cataloguing the archives}

The biggest and most crucial component of the project is the cataloguing of the information in the archives. This has involved most staff of the Cultural Centre and is dependent upon their expertise. As of late 2006, over twenty thousand items from the VCC archives have been catalogued, albeit to varying degrees and not all of which are (as yet) fully integrated into the network.

\section{Digitisation}

Another major area of work is the digitisation of portions of the archives. Digitisation is more important and more appropriate for some formats than others. Museum artefacts, for example, do not lend themselves to digitisation very well, while for photographs, audio and video recordings, digitisation is much easier and more appropriate. As such, our digitisation efforts are focussing primarily on photographic, audio and video recordings in the archives, and on incorporating digital texts where already available.

\section{Software: cataloguing and content management applications}

The VCIN requires computer software to catalogue and retrieve information as well as to manage our digital content. This software must be capable of meeting each of our goals and principles mentioned above. These requirements are unique, particularly our approach to trilingualism and translingualism, our system of tabu restrictions, and our need for a system to manage and integrate our digital files. Moreover, we need a system to do all of this in a way that would be accessible to a wide variety of users.

Typically, commercial cataloguing software packages focus on one particular area: there are library packages, museum packages, photographic archiving packages, but we require a system that does all of this, as well as meeting the other requirements outlined above. At the time that this project was initiated we could not find an appropriate commercial off-the-shelf solution, so we decided we would develop our own applications. 
The software we are developing, our Content Management System (CMS), uses a combination of web technologies, including: Coldfusion (an application development framework), Extensible Hypertext Markup Language (XHTML), Cascading Style Sheets (CSS), JavaScript and Structured Query Language (SQL). The use of these web technologies has the benefit of making the system easily deployable over our internal computer network (our intranet) and suited to future deployment over the internet (via an internet website or as a secure extranet), if desired.

Developing our own software is allowing us to ensure that we get a system that precisely meets our needs, and it will allow us to modify and update that system as we require. However, there are challenges and drawbacks to developing our own software, most notably the time and expertise necessary.

\section{The Vanuatu Cultural Thesaurus}

A fourth area of work has been to develop a thesaurus to be used in cataloguing. A thesaurus is a word or subject list used in describing and categorising items in cataloguing and to assist in generating relevant search results for users. In this case, the aim is to develop a thesaurus that can broadly encompass all relevant areas of culture in Vanuatu and thus, the vast majority of the holdings of the VCC. The majority of the holdings of the Public Library are a notable exception to this. As much of its holdings come from overseas and are not necessarily concerned with Vanuatu, the Public Library uses the Sears List of Subject Headings, an international English-language subject list used by many special libraries. The National Library uses both the Vanuatu Cultural Thesaurus and the Sears List of Subject Headings in cataloguing its Vanuatu and Pacific Collections.

The thesaurus is used to provide additional description to items in a manner that is consistent, so as to allow for users to find all items on a certain topic. As every cataloguer would presumably describe some items differently from another cataloguer, a thesaurus or subject list is intended to standardise description. It provides a controlled vocabulary and represents an agreement among cataloguers on whether to use the term 'family' or 'kinship', for example. But the standard subject lists that are used by libraries internationally are quite large, yet not necessarily appropriate. They contain many terms that are irrelevant to the Cultural Centre, and yet do not contain many words that are crucial to describing cultural knowledge in Vanuatu.

Consequently, we decided to develop our own subject list specific to Vanuatu, but we also needed to make it trilingual, which meant deciding upon appropriate translations and spellings of words. The challenge is in keeping the list as short as is reasonable, so that cataloguers can come to know it very well in a short period of time. Also different words are of importance to different sections of 
the Cultural Centre, so a lot of discussion and compromise has been necessary in the development of the cultural thesaurus. And it is the thesaurus that facilitates the trilingual functioning of the catalogue without each of the items in the collection needing to be fully catalogued in each of the three languages. The Vanuatu Cultural Thesaurus currently contains approximately 250 words in each of the three languages.

\section{Unique features and innovations}

The Vanuatu Cultural Information Network is unique in the Pacific, if not the world for its integration of multiple media and archival institutions, and for its approach to multilingualism and translingualism.

A system such as the VCIN can only be as valuable as the content it promotes and manages. As such, what really makes this initiative unique is what makes the Vanuatu Cultural Centre itself unique: very rarely do you find a nation's primary cultural institutions so well integrated and cooperative at the national level. As a result we are able to create an incredibly rich and valuable resource for Vanuatu.

The project is also unique in being trilingual and in its approach to multi- and translingualism. Not only is the catalogue able to make the most (multilingually) out of the Cultural Centre's limited cataloguing resources, and cater to and take advantage of the multi- and translingual skills of the typical user of the Cultural Centre, but the project also leaves open the possibility of adding additional languages in the future with relative ease. By translating the Cultural Thesaurus into another language, the catalogues would gain basic search functionality in that language.

\section{Role of researchers}

The project should make it much easier for researchers to access the archives, just as it makes it easier for staff, fieldworkers, and the general public. In addition, the project should give researchers a better understanding of the relationships between items in the collections. The system we are creating should also make it easier for researchers to contribute to the archives, both in terms of contributing knowledge and digital files, particularly in the case of photographs.

The potential exists for our photographic archives in particular to grow very quickly. Very simply, it is easier for researchers, fieldworkers, staff, public and tourists to generate digital photographs in much greater number than they 
generate audio or video recordings, or books. So plans for the network include setting up a system whereby, if a researcher, fieldworker, visitor or member of the public comes to the Cultural Centre with photos to contribute, they can be given a password that will allow them to login to the Content Management System, upload their photos, and enter into the catalogue any information they can provide about them. Then the Cultural Centre's photo archivist would only need to review the uploaded photos and the information provided, and add any additional information required, in order for the photos to become part of the archives. This will save the archivist time in data-entry, and takes advantage of the specific knowledge of the collector/photographer.

This has the potential to allow our photographic archives to grow quickly while reducing the resources and expense required by the Cultural Centre. We are also looking to add a similar feature to allow approved staff, researchers, and fieldworkers to add comments to catalogue entries in a style similar to a 'wiki'. Further to this though, we have begun to include more biographical information in the catalogues. As such, if one contributes photographs, for example, we will ask that they provide biographical and contact information about themselves, as well as for the people appearing in their photographs and this information will be linked to all relevant catalogue entries, leading to the creation of a bibliographic database.

\section{Impacts of the project}

\section{Conservation and accessibility through digitisation}

In this project, digitisation is a tool in the conservation of the archives because digital copies (where appropriate): provide backups which are more easily transported off-site for protection against damage from natural disasters, fires and theft; are potentially more easily transferred to new media formats; make the creation of additional backups quicker and easier; can be more easily checked for data integrity; and reduce the need to access the original copies, thus reducing the chance of damage to the originals.

Furthermore, digitisation, and the ease of access to digital records created by the Content Management System (CMS), allow for greater access to the archives by the public. Through the CMS, digital archives can be accessed, where appropriate, without the need for staff or users to access the original items or the rooms which hold them, saving time for both staff and user and protecting the original objects. Further, the VCIN allows for the potential of remote access to the archives in the future. 


\section{Impacts on the ways in which users interact with the archives}

The VCIN makes the archives more accessible to cultural research by helping VKS/VCC staff to locate and access the archives of their section and the archives of other sections of the Vanuatu Cultural Centre with relative ease. The VCIN also permits users to access the archives directly, where appropriate, and it facilitates more detailed documentation of information about the archives and the relations between objects.

However, the VCIN not only changes the way in which VCC staff and users interact with the archives, but also how users interact with VCC staff and with the archives via staff. Such a system makes some information accessible without the need to interact with staff, and thus changes the role of staff as gatekeepers of the archives. Staff still control the level of accessibility of information stored in the archives, but once the appropriate staff member assigns a level of accessibility, then anyone who is granted that level of access can potentially access that information without interacting directly with a staff member. In short, while VCC staff control the CMS, setting the parameters for access to individual objects, some of the role of keeper and gatekeeper of knowledge is transferred from the staff member to the CMS as the user interacts with the CMS to retrieve cultural information, rather than the staff of VCC.

This approach can potentially save VCC staff time in locating and retrieving objects while also ensuring that knowledge is not lost through staff turnover or damage to the archives. However, it also changes the role of the staff member and the dynamic between staff and users changing the transfer of knowledge from an oral to a written process and potentially reduces personal contact between VCC staff and users of the archives. What effect will this have on the relationship between VCC staff and users of the archives? What impacts will any changes in these relationships have on cultural research?

\section{More effective integration of information from multiple media forms from different sections of the Cultural Centre}

The system promises the ability to relate various types of media and information from across the Cultural Centre. Again, this means that users can quickly and efficiently access data from various sources and in various media forms: listening to a radio program, viewing photos and reading cultural information retrieved from newspapers or scanned documents, or in databases included in the Network. 


\section{Making relationships explicit}

The VCIN attempts to make pre-existing relationships among data explicit, drawing connections between objects, catalogue records, place, language and people. These are relations that in some cases would otherwise appear invisible. This is, in effect, a re-contextualisation of objects in the archives into their social, spatial and archival contexts. ${ }^{2}$

\section{Planned and potential future directions}

\section{Disseminating information from our catalogues}

Up until this point, the focus of the project has been on developing the software, developing the cataloguing practices across and within sections of the VCC, cataloguing objects, and making the catalogues useful to relevant staff of VCC.

The next step envisaged is to make portions of these catalogues available to all staff in the Cultural Centre and to visitors to the Cultural Centre. Following this, the next step would be to consider making the catalogues and possibly part of our collections accessible beyond the Cultural Centre. This could be nationally or internationally via the internet or by setting up the catalogues at a few select institutions (offline or using an extranet).

\section{Disseminating software and exchanging information}

A further step would be to exchange collection information with other institutions in Vanuatu in order to expand our collections and allow the VCIN to provide information about holdings elsewhere. This would be particularly useful for the National and Public Libraries to be able to direct users to information held elsewhere and to find out about publications on Vanuatu which are not yet held by the libraries. This would be to make progress toward a broader, national information-sharing network.

One way in which this could be facilitated would be to share the software that we are developing for this project. This would require VCC to support these libraries and provide training and installation assistance. While VCC does not currently have the staffing resources to offer that, there would be a number of advantages to disseminating the software, particularly in facilitating the exchange of information and creating a broader national information sharing network. As such, this is a potential future direction for VCC and the VCIN.

2 For a detailed analysis of the links between digital and social relationships in the VCC database, see Geismar and Mohns, (2011). 


\section{Costs of supporting and expanding the VCIN}

Another important element to consider is the cost involved in maintaining the system and in continually adding content to the VCIN. At the same time, it is important to recognise that good electronic digital content-which the Cultural Centre definitely has - is a valuable commodity. As such, an important consideration in future directions may be to assess the potential for recovering some of the costs of providing services at the national or international level and to expanding the scope of the project. A further question is whether or not there is interest in making portions of our digital archive available overseas, via the internet or otherwise.

\section{Requirements for success}

The project is currently at a crucial stage in its development, and its continued success will depend upon substantial investment in the development of VCC's in-house expertise in electronic cataloguing, digitisation, and web development.

\section{Final thoughts}

The Vanuatu Cultural Information Network is an ambitious and groundbreaking project of the Vanuatu Cultural Centre. A great deal has been accomplished already, particularly in the cataloguing of the Cultural Centre archives, and the development of a unique Content Management System to run the Information Network. The results of the VCIN are greater accessibility and therefore increased use of the Vanuatu Cultural Centre Archives; increased knowledge stored in the archives; and an increase in the security of the archives. Yet a great deal of work remains on the project, and there is considerable flexibility in what directions the project will go and how much of its potential it will be able to achieve.

\section{References}

Geismar, Haidy and William Mohns, 2011. Social relationships and digital relationships: rethinking the database at the Vanuatu Cultural Centre. In The Aesthetics of Nations: Anthropological and Historical Approaches. Special Issue of the Journal of the Royal Anthropological Institute, vol. 17, issue supplement sl: 133-55, ed. Christopher Pinney and Nayanika Mookherjee. 